\title{
Legislación y Normas Atingentes a la Atención Odontológica y el COVID-19. Una Perspectiva desde las Clínicas Universitarias
}

\author{
Lesgislation and Norms Related to Dental Care and the COVID-19. \\ A Perspective from University Clinics
}

Ramón Fuentes ${ }^{1}$ Carlos Zaror ${ }^{2} \&$ Marcela Huanquilef ${ }^{1}$

FUENTES, R.; ZAROR, C. \& HUANQUILEF, M. Legislación y normas atingentes a la atención odontológica y el COVID19. Una perspectiva desde las clínicas universitarias. Int. J. Odontostomat., 14(4):481-488, 2020.

RESUMEN:La enfermedad por coronavirus 2019 (COVID-19) es una enfermedad infecciosa causada por el Coronavirus 2 del Síndrome Respiratorio Agudo Grave (SARS-CoV-2). La mayoría de los pacientes infectados con SARSCoV-2 tiene una evolución asintomática o leve. Sin embargo, una parte de los infectados presentará un deterioro en su condición de salud, desarrollando una neumonía aguda grave, que puede producir la muerte. Las personas vinculadas a las clínicas odontológicas están en riesgo de infección por SARS-CoV-2, pues la saliva es un importante método de transmisión del virus. Por su naturaleza, la atención odontológica se realiza a corta distancia del paciente, con constante exposición a saliva, sangre y otros fluidos corporales, y es frecuente el manejo de instrumentos cortopunzantes. Por consiguiente, los elementos de protección personal cobran gran importancia y las reglamentaciones de la autoridad sanitaria en el contexto de la pandemia que actualmente se vive deben cumplirse a cabalidad, debido a obligaciones éticas y legales. En esta revisión narrativa se revisa y discute la reglamentación de la autoridad sanitaria que involucra las prestaciones odontológicas en el contexto del COVID-19, cómo se aplicará en las clínicas universitarias y cómo complementar la normativa con la evidencia científica disponible.

PALABRAS CLAVE: COVID-19, Normativa atención Odontológica, clínicas Universitarias.

\section{INTRODUCCIÓN}

La enfermedad por coronavirus 2019 (COVID19) es una enfermedad infecciosa causada por el Coronavirus 2 del Síndrome Respiratorio Agudo Grave (SARS-CoV-2) (Rothan \& Byrareddy, 2020). EI SARS-CoV-2 tiene un origen animal (Rothan \& Byrareddy), aunque actualmente se transmite de persona a persona, lo que explica en parte su alta tasa de contagio. Los primeros casos de síntomas de neumonía de origen desconocido se han logrado rastrear hasta el 1 de diciembre de 2019 en Wuhan, China (Helmy et al., 2020; Lake, 2020), por lo que la transmisión animal-humano del virus ocurrió probablemente en noviembre de dicho año. El 7 de enero de 2020 se logró aislar y decodificar el genoma del nuevo virus (Chan et al., 2020), llamado inicialmente como 2019nCoV por la Organización Mundial de la Salud (OMS, 2020a), mientras se reportaba un aumento diario de casos. No fue hasta el 23 de enero que las autoridades chinas declararon el cierre de la ciudad de Wuhan, pero para aquel entonces el virus se había ya propagado a otras regiones chinas y a países vecinos (OMS, 2020a; Helmy et al.). Actualmente casi la totalidad de los países del mundo han presentado diagnósticos de COVID-19 y el número de fallecidos a nivel global alcanza los 284.792 para el 11 de mayo de 2020 .

La mayoría de los pacientes infectados con SARS-CoV-2 tiene una evolución asintomática o leve (Arashiro et al., 2020; Chen et al., 2020), con síntomas inespecíficos que incluyen fiebre, tos seca, dolor de cabeza y malestar general. Sin embargo, una parte de los infectados presentará un deterioro en su

\footnotetext{
${ }^{1}$ Centro de Investigación en Ciencias Odontológicas (CICO), Facultad de Odontología, Universidad de La Frontera, Temuco, Chile.

${ }^{2}$ Departamento de Odontopediatría, Facultad de Odontología, Universidad de La Frontera, Temuco, Chile.
} 
condición de salud, desarrollando una neumonía aguda grave, que requiere cuidados intensivos, conexión a ventilación mecánica y que puede incluso producir la muerte (Chen et al.). La autopsia de pacientes fallecidos por COVID-19 revela también pericarditis (Hanley et al., 2020). En un intento de frenar el número de contagios y evitar el colapso de los sistemas de salud, se ha paralizado las actividades no esenciales, cerrado las fronteras, comercios y gran parte de las actividades aeroportuarias, y prohibido la reunión de personas, lo que ha afectado de manera significativa la actividad económica y ha llevado a algunos países a una recesión sin comparación con ninguna crisis económica previa.

Las vías de transmisión del SARS-CoV-2 incluyen la transmisión directa, como tos, estornudos y por inhalación de gotas, y transmisión por contacto, como el contacto con las membranas mucosas orales y nasales, y puede darse desde una persona sintomática como asintomática (Rothe et al., 2020). Así mismo, el virus puede permanecer viable en aerosoles por horas (van Doremalen et al., 2020) y la exposición ocular puede proporcionar una forma efectiva para que el virus ingrese al cuerpo.

El primer diagnóstico de contagio de COVID-19 fue reportado en Chile el 3 de marzo de 2020, aunque actualmente se sospecha, como en otros países, que el primer contagio ocurrió con anterioridad y no fue diagnosticado en su momento. Es altamente probable que las fechas de ocurrencia de los primeros casos de COVID-19 en Chile y en otros países varíen al reanalizar casos de neumonías sin causa aparente que ocurrieron antes de que estallara la crisis de manera pública. Según datos oficiales del Gobierno de Chile (2020), al 11 de mayo de 2020 se han realizado 294.057 exámenes de PCR, hay 30.063 contagiados y han fallecido 323 personas, lo que entrega una tasa de mortalidad del $0.93 \%$, una de las más baja entre los países de la OCDE. Sin embargo, se debe considerar que la tasa de contagio crece de manera exponencial.

Actualmente todas las regiones presentan casos positivos de SARS-CoV-2. Sin embargo, se observan grandes diferencias por región. Las regiones más afectadas son la Metropolitana de Santiago (21.317 casos), la Araucanía (1.466), Antofagasta (1014) y Magallanes (901), y las menos afectadas son la región de Atacama (133 casos) y Aysén, con apenas 8 casos para el 11 de mayo de 2020. Se ha dictado cuarentena y cordón sanitario diferenciada para las comunas más afectadas, con duraciones variables. Se sospecha que las regiones del sur chileno se verán especialmente afectadas debido a las peores condiciones climáticas del invierno entrante, a la disminución en las horas de sol que causa disminución de síntesis de vitamina D (Marik et al., 2020), y a la importante polución por material particulado (Sciomer et al., 2020), como la producida por el uso de la leña como método de calefacción. Según se ha demostrado el material particulado está relacionado con el empeoramiento de la presentación clínica de infección por virus en las vías respiratorias (Carugno et al., 2016).

Las personas vinculadas a las clínicas odontológicas, incluyendo a odontólogos, estudiantes de odontología, docentes de las Clínicas Docente Asistenciales, personal auxiliar, pacientes y acompañantes están en riesgo de infección por SARS-CoV-2 y otras enfermedades infectocontagiosas. Por su naturaleza, la atención odontológica se realiza a corta distancia del paciente, con constante exposición a saliva, sangre y otros fluidos corporales, y es frecuente el manejo de instrumentos cortopunzantes. En consecuencia y como método de prevención de contagio, la odontológica y demás ciencias médicas han desarrollado elementos de protección personal (EPP), que constituyen la última barrera que protege al prestador de salud contra potenciales daños a la salud por exposición a diversos agentes infectocontagiosos (Honda \& Iwata, 2016).

El objetivo de esta revisión narrativa es revisar y discutir las reglamentaciones de la autoridad sanitaria que son atingentes a las prestaciones odontológicas en el contexto del COVID-19, cómo se aplicará en las clínicas universitarias y cómo complementar la normativa con la evidencia científica disponible.

\section{LEGISLACIÓN Y REGULACIÓN VIGENTE}

Las regulaciones de la autoridad sanitaria para el ejercicio de la odontología deben cumplirse sobre todo en el contexto del COVID-19, pues es el marco legal mínimo que rige el ejercicio de la profesión. La legislación nacional establece que todo establecimiento que brinde atención odontológica, incluyendo las clínicas universitarias, deben tener su autorización sanitaria vigente, que emite la Secretaría Regional Ministerial (SEREMI) correspondiente. La Acreditación para Prestadores Institucionales de Salud es de carácter voluntario y está regulado por la Intendencia de Prestadores Institucionales de Salud (Superintendencia de Salud, 2020). 
Las regulaciones en atención de salud y odontológicas establecidas por la autoridad sanitaria y que tienen relevancia para el contexto del COVID19 son las siguientes:

1.- Circular C13 No19 del año 2013 del Ministerio de Salud, que establece precauciones estándar para el control de infecciones en la atención en salud y algunas consideraciones sobre aislamiento de pacientes (Ministerio de Salud, 2013). Esta circular establece normas para la higiene de manos, el uso de guantes, protección facial, delantal, manejo de material cortopunzante, higiene respiratoria y buenos hábitos al toser y estornudar, el equipamiento clínico para el cuidado del paciente, aislamiento de pacientes con diagnóstico o sospecha de diagnóstico de enfermedad contagiosa y la técnica aséptica, que establece el uso de material estéril cada vez que se realice un procedimiento invasivo. Esta circular establece que los establecimientos de salud deben proveer acceso a los insumos, equipamiento e instalaciones, además capacitar al personal para el correcto cumplimiento de las instrucciones. Ante brotes infecciosos, como el que se vive actualmente con el SARS-CoV-2, la circular establece que se deben actualizar las capacitaciones y preparación para cumplir con la circular (Ministerio de Salud, 2013).

2.- La Subsecretaria de Redes Asistenciales a través de la Circular Circular C37 No2 ha dictado la Racionalización uso EPP en contexto atención pacientes durante pandemia de COVID-19. Esta circular establece que los directores de los servicios y establecimientos de salud deben designar a un encargado para velar por el correcto uso de elementos de protección personal, así como velar por su inventario. Establece la priorización del uso de EPP, su uso extendido y su desinfección para reutilización (Subsecretaria de Redes Asistenciales, 2020a).

3.- Norma General Técnica №199 sobre esterilización y desinfección en establecimientos de atención de salud (Ministerio de Salud, 2018a). Esta norma hace referencia a los principios del correcto uso de artículos médicos estériles y procesos de esterilización y desinfección de alto nivel para prestadores de salud. Los artículos médicos deben clasificarse según el nivel de eliminación de microorganismos que requieran, para luego proceder a la esterilización/desinfección correspondiente según los métodos descritos por la Norma (Ministerio de Salud, 2018a).

4.- Circular C37 №10 del año 2018 sobre el aseo y desinfección de superficies ambientales para la prevención de infecciones asociadas a la atención en salud (Circular C37,Ministerio de Salud, 2018b). Esta Norma define las superficies ambientales como aquellas que normalmente no tienen contacto directo con el paciente o que tienen un contacto menor e incluyen superficies de equipos médicos y superficies domésticas (suelo, muros, manillas, etc.). Estas superficies deben ser primero limpiadas y luego desinfectadas para disminuir la carga microbiana en estas y reducir el riesgo de contagio. La Norma define distintos métodos de desinfección y supervisión de dicho proceso. Finalmente, la Norma recalca que el aseo y desinfección de superficies ambientales previene infecciones por agentes específicos como el coronavirus responsable del SARS (SARS-CoV-1), que causó un brote epidemiológico en el sureste asiático en 2003 (Ministerio de Salud, 2018b)

5.-La Subsecretaria de Salud Pública a través de la División de Prevención y Control de Enfermedades y su Departamento de Salud Bucal entregaron Orientaciones para la Atención Odontológica en Fase IV de COVID-19 (Subsecretaría de Salud Pública, 2020). Estas orientaciones tienen por objetivo el establecer recomendaciones específicas para el personal de salud bucal que entrega atención odontológica a casos sospechosos o confirmados de COVID-19, determinar la conducta a seguir de acuerdo al motivo de la consulta odontológica espontánea o programada de casos sospechosos o confirmados de COVID-19 y entregar recomendaciones para garantizar la continuidad de la atención odontológica de casos sospechosos o confirmados de COVID-19 (Subsecretaría de Salud Pública).

6.- La Subsecretaria de Redes Asistenciales a través de la Circular $\mathrm{N}^{\circ} 005$ ha publicado el Protocolo de reprocesamiento de respiradores tipo N95, FFP2 u otros equivalentes, para atención clínica en el contexto de pandemia COVID-19, donde se establece la posibilidad de reutilizar los respirados N95 tras su desinfección con vapor o plasma de Peróxido de Hidrógeno, en casos de que no se encuentre con stock local de respiradores para 30 días o menos y no hay seguridad de abastecimiento para asegurar la continuidad de la atención y seguridad del equipo de salud (Subsecretaria de Redes Asistenciales, 2020b).

Adicional a estas Normas y Circulares, el Ministerio de Salud está constantemente publicando Normas y Resoluciones Exentas respecto a la alerta y refuerzo de vigilancia epidemiológica ante brote del SARS-CoV-2 y pueden ser vistas en el sitio WEB del Ministerio de Salud (Ministerio de Salud, 2020). 
Regionalmente La Secretaría Regional Ministerial de la Araucanía dictó la Resolución Exenta $N^{\circ}$ J1- 1649 y la Secretaría Regional Ministerial de Nuble dictó la Resolución Exenta № 8921 de del que prohíben las atenciones odontológicas en las comunas de Temuco y Padre las Casas y en la Región del Ñuble (Secretaría Regional Ministerial de Salud del Nuble, 2020), respectivamente, salvo aquellas calificadas de urgencia, debiendo reprogramarse cualquier otro tipo de atención dental. Lo anterior paraliza cualquier tratamiento dental que no sea de urgencia en clínicas privadas, establecimientos públicos y clínicas universitarias. En los Vistos de La Resolución Exenta №J1-1649 se lee "en los vistos "Presentación de fecha 01.04.2020 de la Mesa Directiva del Colegio de Cirujanos Dentistas de Chile A.G." Llama la atención este punto. No tenemos antecedentes que se haya solicitado en otras regiones y comunas y a nuestro juicio el Colegio actuó sin la prudencia adecuada y sin consultar con regionalmente a los representantes de la Odontología. Con fecha 20 de Mayo de 2020, la Seremi de la Araucanía dicta la Resolución Exenta J1002580, que deja sin efecto la resolución antes emitida y resuelve que en las atenciones odontológicas se deberá dar estricto cumplimientos a las orientaciones impartidas por la Subsecretaría de Salud Pública para la Atención Odontológica en Fase IV COVID-19.

\section{ROL DE LAS CLÍNICAS UNIVERSITARIAS EN LA PANDEMIA}

Rol Científico: EI COVID-19 es una situación emergente, con comportamiento dinámico y evolución no conocida a cabalidad. Por consiguiente, se están realizando un número exponencial de experimentos y publicaciones en la comunidad científica en un plazo muy reducido de tiempo. Las clínicas universitarias tienen como marco regulatorio las normas, resoluciones y orientaciones de la autoridad sanitaria antes mencionadas, pero se debe considerar que es probable que el rápido ritmo de experimentos produzca conocimiento nuevo, que tarde en incorporarse en las normas y resoluciones oficiales. Por consiguiente, los equipos técnicos de las universidades deben estar continuamente actualizándose con la última evidencia científica disponible para proponer, de ser necesario, complementos a las normativas vigentes, especialmente en relación con la atención en las clínicas universitarias.

El proceso de elaboración de un protocolo clínico basado en evidencia requiere de una metodolo- gía rigurosa que asegure que las recomendaciones entregadas tengan una base científica. Una vez definidos el alcance, objetivos y preguntas clínicas, se debe hacer una búsqueda de las guías y protocolos existentes con el fin de evaluar la factibilidad de adaptar uno existente o de realizar uno nuevo. La calidad de estas guías debe ser evaluada a través del instrumento AGREE (Appraisal of Guidelines Research \& Evaluation) (Brouwers et al., 2020) antes de utilizar las recomendaciones entregadas por estas. En el caso de no existir evidencia que sustente las recomendaciones se deben realizar revisiones sistemáticas específicas para cada pregunta y luego evaluar la calidad del cuerpo de la evidencia a través de la metodología GRADE (Grades of Recommendation, Assessment, Development, and Evaluation) (Guyatt et al., 2008).

Rol de Empleador: las instituciones universitarias tienen un rol de empleador y deben asegurar que el personal ejerza sus funciones bajo el cumplimiento normativo, es decir, bajo las leyes laborales y normas chilenas de seguridad y bioseguridad. Este cumplimiento se debe coordinar con la dirección de personal correspondiente y la mutualidad adscrita para facilitar su adecuada implementación. El uso de elementos de protección personal cobra gran relevancia en el quehacer laboral del personal docente y auxiliar, dada la naturaleza y contexto de las probabilidades de contagio y de enfermar en la atención odontológica. Los elementos de protección personal presuponen ser utilizados en un individuo sano o con salud compatible para la ocupación y se debe evaluar la necesidad de agregar barreras o medios para proteger al operador frente el agente infeccioso14, en este caso el SARS-CoV-2. Adicionalmente, como establece la circular C13 No19 del año 2013 del Ministerio de Salud, es función de las clínicas universitarias el proveer acceso a los insumos, equipamiento e instalaciones, además capacitar al personal para el correcto cumplimiento de las instrucciones para el control de infecciones en la atención en salud. Esto obviamente llevará a nuevos gastos por parte de los sistemas clínicos.

Rol Docente: Las unidades académicas y sus respectivas asignaturas deben actualizar la entrega de conocimiento y educar a los estudiantes de pregrado y postgrado sobre cómo los elementos de protección personal desechables y/o reutilizables son reconocidos como obligatorios por las normas chilenas de seguridad para la ejecución de un acto clínico, además de la actualización científica sobre su 
correcto uso y cómo evitar el contagio. En el contexto de la pandemia que actualmente se vive, es recomendable que dicha actualización y reforzamiento se dé en todos los niveles teóricos de la carrera de pregrado y especialidades, y que exista un reforzamiento y vigilancia continua por parte del docente instructor durante el quehacer práctico.

Rol Sanitario: Para el desarrollo de una atención de urgencia o aquella que no se pueda diferir, como orienta el MINSAL en la Fase IV COVID-19, es necesario implementar un plan de abordaje, donde la normativa y las orientaciones se cumplan rigurosamente mediante la aplicación de instructivos y protocolos que describan los flujos del sistema de atención de pacientes y sus rutinas de aseguramiento en bioseguridad.

\section{ATENCIÓN ODONTOLÓGICA CON Y SIN GENERA- CIÓN DE AEROSOLES Y ELEMENTOS DE PRO- TECCIÓN PERSONAL}

Los elementos de protección personal que incluyen las Orientaciones para la Atención Odontológica en Fase IV COVID-19 del Ministerio de Salud (Subsecretaría de Salud Pública) se analizan a continuación según el tipo de atención odontológica.

Atención sin generación de aerosoles. Los elementos de protección personal que debe utilizar el equipo odontológico que preste atención clínica sin procedimientos generadores de aerosoles incluyen:

a) Guantes desechables e impermeables de látex o nitrilo.

b) Mascarilla quirúrgica o de procedimiento.

Ambos elementos han sido de uso habitual en el ejercicio clínico odontológico bajo normativa previa al COVID-19 (Minsal, 2013a).

c) Protección ocular que incluye el uso de antiparras o escudo facial. Las antiparras deben ser de material impermeable que no se empañe; deben cubrir los ojos y las áreas circundantes alrededor del marco de las antiparras; deben permitir el uso de lentes ópticos y poder ser retiradas sin remover los lentes ópticos.

d) Bata o delantal de manga larga y apertura posterior, impermeable y desechable.

Ambos elementos (protección ocular y delantal) se agregaron en las Orientaciones para la Atención Odontológica en Fase IV del COVID-19 (Subsecretaría de Salud Pública) para el equipo odontológico profesional y asistente dental clínico.

Atención con generación de aerosoles. Los elementos de protección personal que debe utilizar el equipo odontológico que preste atención clínica con procedimientos generadores de aerosoles incluyen:

a) Guantes desechables e impermeables de látex o nitrilo.

b) Respirador tipo N95, FFP2 o equivalente.

c) Protección ocular similar a la descrita anteriormente

d) Bata o delantal de manga larga y apertura posterior, impermeable y desechable.

Los últimos tres elementos se agregan a través de las Orientaciones para la atención odontológica en Fase IV del COVID-19 para el equipo odontológico profesional y asistente dental clínico (Subsecretaría de Salud Pública).

Como sistema clínico universitario recomendamos para ambas atenciones el uso de gorro desechable, la racionalización de los elementos de protección personal basado en evidencia respecto a vida útil y uso efectivo del uniforme clínico completo.

Los elementos de protección personal también se encuentran categorizados según el Listado de Normas Chilenas Área C. 9: Protección Personal según Segmento Corporal (Instituto Nacional de Normalización, 2008).

Elementos para la protección de ojos y cara:

- Antiparras con condición antiempañables.

- Escudo facial antiempañables.

Elementos para la protección de las vías respiratorias:

- Respirador tipo N95 o FFP2 o su equivalente

Elementos de protección de manos y brazos:

- Guantes desechables, impermeables, de látex o nitrilo y con puño.

Elementos de protección con ropa protectora: - Bata manga larga impermeable y desechable - Delantal manga larga impermeable y desechable.

Respeto a las ventajas y limitaciones que presentan los elementos de protección se encuentran:

Ventajas: Rapidez en su implementación y fácil de usar. Antes de la pandemia también destacaba su disponibilidad en el mercado y bajo costo.

Limitaciones: Falsa sensación de seguridad. Los elementos de protección solo reducen el riesgo, pero no lo eliminan. Adicionalmente, requieren de manteni- 
miento riguroso y periódico, una implementación a largo plazo o sostenida en el tiempo, involucran procesos de mantenimiento y reposición, requieren de personal para su supervisión, capacitación y reforzamiento y manipulación correcta.

La adopción de buenas prácticas en el manejo de insumos reciclables o de reutilización, no necesita un estudio de factibilidad detallada, ya que su implementación solo requiere la voluntad de la institución empleadora y no la aplicación de modificaciones tecnológicas, según establece la Subsecretaria de Salud Pública Chilena. Extender la vida útil de los elementos de protección personal dependerá de su materialidad y propiedades para ser limpiados o esterilizados, según corresponda.

Los elementos de protección personal que no pierden o no ven alteradas sus propiedades de protección, como lo son las antiparras y los arcos faciales recomendados por el Ministerio de Salud chileno, deben ser lavados siguiendo un instructivo de limpieza. Los insumos reciclables o de reutilización deben ser llevados a una línea de lavado para limpieza por arrastre con solución de agua jabonosa. Para la limpieza, el área de trabajo debe considerar áreas sucias, limpias, secado y almacenaje.

La eliminación de los residuos de establecimientos de atención en salud (REAS) debe cumplir el Manejo Interno de REAS Punto 4.1 páginas 31 a 40, que se describe paso a paso en el Manual de Residuos de Establecimientos de Atención de Salud, de la Subsecretaría de Salud Pública (Ministerio de Salud, 2010). Debido al alto número de estudiantes y profesionales en una clínica universitaria, especial importancia cobra la implementación de una rutina para el correcto retiro y eliminación de los elementos de protección personal desechables antes de la eliminación de los residuos propios de esta atención clínica, como lo establece la norma.

\section{ESPACIOS ADMINISTRATIVOS EN UNA CLÍNICA ODONTOLÓGICA UNIVERSITARIA}

De acuerdo con las Orientaciones del Ministerio de Salud para la Atención Odontológica en Fase IV Covid-19 (Subsecretaría de Salud Pública) se deben implementar elementos de protección personal y rutinas de acceso a las instalaciones del personal administrativo. Los espacios comunes como sala de esperas, áreas de circulación, recibidor de acceso y baños deberán estar demarcados para que se cumpla con el distanciamiento de social de un metro.

\section{PACIENTES DE LAS CLINICAS ODONTOLOGIAS}

Diversos estudios han demostrado que el SARS-CoV-2 puede sobrevivir hasta 72 horas en superficies de plástico y acero inoxidable, menos de 4 horas en superficies de cobre y menos de 24 horas en superficies de cartón (OMS, 2020b). No está claro cuándo tiempo sobrevive el virus en el asfalto, pero ante la posibilidad de que los zapatos estén contaminados con el virus se deben tomar medidas desde el ingreso de personas a la clínica y desinfectar, a lo menos, la planta del calzado. Es importante no implementar medidas que no cuenten con la suficiente evidencia o que estén en cuestionamiento ya sea por su potencial falta de efectividad o por su posibilidad de toxicidad, como por ejemplo los túneles sanitarios.

Se requerirá implementar un cuestionario de cuadro clínico que considere signos y síntomas asociados a la enfermedad COVID-19, ya sea en un primer contacto telefónico o presencialmente en la consulta en la mesa de ayuda y previo a la atención. Se recomienda que los pacientes con sintomatología sugerente de contagio por SARS-CoV-2 sean derivados a evaluación médica; cada clínica universitaria puede definir si prestará atención a pacientes COVID-19 (+) o implementará una red de derivación fuera de la institución.

Es aconsejable instruir a los pacientes sobre sus derechos y deberes y, en lo que al paciente concierne, sobre las Orientaciones Ministeriales para la atención odontológica en Fase IV COVID-19 (Subsecretaría de Salud Pública).

\section{CONCLUSIONES}

Existen numerosas Normas y regulaciones de la autoridad sanitaria para la protección de personas asociadas a la atención odontológica. Estas normas deben cumplirse, pues es un deber ético y legal.

Para poder realizar una atención odontológica en el contexto de la pandemia de COVID-19 deberá existir disponibilidad de insumos y elementos de protección personal. El alto valor económico actual de los elementos de protección personal y la falta de stock serán las limitaciones que deberemos abordar como sistemas universitarios.

Se requerirá una adecuada y permanente formación instruccional sobre las orientaciones y norma- 
tivas vigentes en todos los actores de la atención odontológica del sistema clínico universitario.

Se debe poner atención al impacto que esta pandemia y sus consecuencias han generado y generaran en la salud mental y estado psicológico de los funcionarios, estudiantes, docentes y pacientes de la clínica odontológica, con el objetivo de generar medidas de prevención y mitigación.

Se recomienda que las clínicas universitarias que deseen complementar las orientaciones ministeriales en FASE IV COVID-19 y las que el ministerio más adelante genere, las formalicen ante la Secretaria Regional Ministerial de Salud correspondiente.

En su rol científico las clínicas universitarias deben analizar cuidadosamente, basado en la evidencia disponible, la implementación de modificaciones semiestructurales en los boxs de atención y de la compra o implementación de equipamiento como los suctores para aerosoles.

Al retomar las actividades clínicas de pregrado y postgrado se deberá analizar, en base a las condiciones sanitarias y normativas, las potenciales modificaciones a los programas de asignaturas y los requisitos clínicos que le exigen a los estudiantes para su aprobación.

Cuando se pueda retomar las atenciones programadas se deberá analizar la implementación de rutinas que los supervisores o instructores clínicos instauren en el uso de los elementos de protección personal en el programa de asignatura.

Por último, se recomienda un esfuerzo comunicacional para dar a conocer las condiciones de bioseguridad adoptadas y basadas en la normativa sanitaria, que permiten una atención con el mayor resguardo sanitario posible.

FUENTES, R.; ZAROR, C. \& HUANQUILEF, M. Legislation and norms related to dental care and the COVID-19. A perspectiv from university clinics. Int. J. Odontostomat., 14(4):481-488, 2020.

ABSTRACT: Coronavirus disease 2019 (COVID-19) is an infectious disease caused by Coronavirus 2 of Severe Acute Respiratory Syndrome (SARS-CoV-2). The majority of patients infected with SARS-CoV-2 have an asymptomatic or mild evolution. However, a part of those infected will present a deterioration in their health condition, developing severe acute pneumonia, which can lead to death. People linked to dental clinics are at risk of SARS-CoV-2 infection, since saliva is an important method of transmission of the virus. Due to its nature, dental care is performed at a short distance from the patient, with constant exposure to saliva, blood and other body fluids, and the use of sharp instruments is frequent. Consequently, the elements of personal protection are very important and the regulations of the health authority in the context of the current pandemic must be fully complied with, due to ethical and legalobligations. This narrative review reviews and discusses the regulation of the health authority that involves dental services in the context of COVID-19, how it will be applied in university clinics and how to complement the regulations with the available scientific evidence.

KEY WORDS: COVID-19, Dental care regulations, University clinics.

\section{REFERENCIAS BIBLIOGRÁFICAS}

Arashiro, T.; Furukawa, K. \& Nakamura, A. COVID-19 in 2 persons with mild upper respiratory symptoms on a cruise ship, Japan. Emerg. Infect. Dis., 26(6):1345-8, 2020.

Brouwers, M. C.; Kho, M. E.; Browman, G. P.; Burgers, J. S.; Cluzeau, F.; Feder, G.; Fervers, B.; Graham, I. D.; Grimshaw, J. Hanna, S. E.; et al. AGREE II: advancing guideline development, reporting and evaluation in health care. CMAJ, 182(18):E839-42, 2020.

Carugno, M.; Consonni, D.; Randi, G.; Catelan, D.; Grisotto, L.; Bertazzi, P. A.; Biggeri, A. \& Baccini, M. Air pollution exposure, cause-specific deaths and hospitalizations in a highly polluted italian region. Environ. Res., 147:415-24, 2016.

Chan, J. F. W.; Kok, K. H.; Zhu, Z.; Chu, H.; To, K. K. W.; Yuan, S. \& Yuen, K. Y. Genomic characterization of the 2019 novel humanpathogenic coronavirus isolated from a patient with atypical pneumonia after visiting Wuhan. Emergi. Microbes Infect., 9(1):221-36, 2020.

Chen, N.; Zhou, M.; Dong, X.; Qu, J.; Gong, F.; Han, Y.; Qiu, Y.; Wang, J.; Liu, Y.; Wei, Y.; Xia, J.; Yu, T.; Zhang, X. \& Zhang, L. Epidemiological and clinical characteristics of 99 cases of 2019 novel coronavirus pneumonia in Wuhan, China: a descriptive study. Lancet, 395(10223):507-13, 2020.

Gobierno de Chile. Cifras Oficiales COVID-19. Santiago de Chile, Gobierno de Chile, 2020. Disponible en: https://www.gob.cl/ coronavirus/cifrasoficiales/

Guyatt, G. H.; Oxman, A. D.; Vist, G. E.; Kunz, R.; Falck-Ytter, Y.; Alonso-Coello, P.; Schünemann, H. J. \& GRADE Working Group. GRADE: an emerging consensus on rating quality of evidence and strength of recommendations. BMJ, 336(7650):924-6, 2008.

Hanley, B.; Lucas, S. B.; Youd, E.; Swift, B. \& Osborn, M. Autopsy in suspected COVID-19 cases. J. Clin. Pathol., 73(5):239-42, 2020.

Helmy, Y. A.; Fawzy, M.; Elaswad, A.; Sobieh, A.; Kenney, S. P. \& Shehata, A. A. The COVID-19 pandemic: a comprehensive review of taxonomy, genetics, epidemiology, diagnosis, treatment, and control. J. Clin. Med., 9(4):E1225, 2020.

Honda, H. \& Iwata, K. Personal protective equipment and improving compliance among healthcare workers in high-risk settings. Curr. Opin. Infect. Dis., 29(4):400-6, 2016.

Instituto Nacional de Normalización. Listado de Normas Chilenas Area C. 9: Protección Personal. Santiago de Chile, Instituto Nacional de Normalización, 2008. Disponible en: http:// 
www.sigweb.cl/wp-content/uploads/biblioteca/ NormasINNEPP.pdf

Lake, M. A. What we know so far: COVID-19 current clinical knowledge and research. Clin. Med. (Lond.), 20(2):124-7, 2020.

Marik, P. E.; Kory, P. \& Varon, J. Does vitamin D status impact mortality from SARS-CoV-2 infection? Med. Drug Discov., 2020. DOI: https://www.doi.org/10.1016/j.medidd.2020.100041

Ministerio de Salud (MINSAL). Manejo de Residuos de Establecimientos de Atención de Salud. Santiago de Chile, Ministerio de Salud, Gobierno de Chile, 2010. Disponible en: http:// eticayseguridad.uc.cl/documentos/comite-seguridad/normativaseguridad/137-dcto-6-reas/file.html.

Ministerio de Salud (MINSAL). Norma General Técnica N¹99 sobre Esterilización y Desinfectación de Alto Nivel y Uso de Artículos Médicos Estériles en Establecimientos de Atención de Salud. Santiago de Chile, Ministerio de Salud, Gobierno de Chile, 2018a. Disponible en:http://www.minsal.cl/wp-content/uploads/ 2018/03/Norma-General-Técnica-Nº-199-sobre-esterilización-ydesinfección-de-alto-nivel-y-uso-de-artículo-médicosestériles.pdf

Ministerio de Salud (MINSAL). Recomendaciones sobre el Aseo y Desinfección de Superficies Ambientales para la Prevención de Infecciones Asociadas a la Atención en Salud. Santiago de Chile, Ministerio de Salud, Gobierno de Chile, 2018b. Disponible en: https://www.minsal.cl/wp-content/uploads/2019/03/Circular$\mathrm{N}$-10-2019-Recomendaciones-sobre-Aseo-y-Desinfección-deSuperficies-Ambientales-para-la-Prevención-de-IAAS.pdf.

Ministerio de Salud (MINSAL). Precauciones Estándar para el Control de Infecciones en la Atención en Salud y Algunas Consideraciones sobre Aislamiento de Pacientes. Santiago de Chile, Ministerio de Salud, Gobierno de Chile, 2013. Disponible en: h t t p s: // w w w. minsal. cl/portal/url/ite m / d8615b8fdab6c48fe04001016401183d.pdf

Ministerio de Salud (MINSAL). Información Técnica. Santiago de Chile, Ministerio de Salud, Gobierno de Chile, 2020. Disponible en: https://www.minsal.cl/nuevo-coronavirus-2019-ncov/informetecnico/

Organización Mundial de la Salud (OMS). COVID-19: Cronología de la Actuación de la OMS. Ginebra, Organización Mundial de la Salud, 2020a. Disponible en: https://www.who.int/es/newsroom/detail/27-04-2020-who-timeline---covid-19

Organización Mundial de la Salud (OMS). Preguntas y Respuestas sobre la Enfermedad por Coronavirus (COVID-19). Ginebra, Organización Mundial de la Salud, 2020b. Disponible en: https:/ /www.who.int/es/emergencies/diseases/novel-coronavirus-2019/ advice-for-public/q-a-coronaviruses

Rothan, H. A. \& Byrareddy, S. N. The epidemiology and pathogenesis of Coronavirus Disease (COVID-19) outbreak. J. Autoimmun., 109:102433, 2020.

Rothe, C.; Schunk, M.; Sothmann, P.; Bretzel, G.; Froeschl, G.; Wallrauch, C.; Zimmer, T.; Thiel, V.; Janke, C.; Guggemos, W.; et al. Transmission of 2019-nCoV infection from an asymptomatic contact in Germany. N. Engl. J. Med., 382(10):970-1, 2020.

Sciomer, S.; Moscucci, F.; Magrì, D.; Badagliacca, R.; Piccirillo, G. \& Agostoni, P. SARS-CoV-2 spread in Northern Italy: what about the pollution role. Environ. Monit. Assess., 192(6):325, 2020.

Secretaría Regional Ministerial de Salud del Ñuble. Resolución Exenta 8921/2020. Chillán, Secretaría Regional Ministerial de Salud del Ñuble, Ministerio de Salud, Gobierno de Chile, 2020. pp.1820. Disponible en: https://www.seremidesaludnuble.cl/wpcontent/uploads/2020/03/RES-EXENTA-8921-2020-Diponemedidas-sanitarias-ALERTA.pdf

Subsecretaria de Redes Asistenciales. Circular C37 No2 Racionalización uso EPP en Contexto Atención Pacientes durante Pandemia de COVID-19. Santiago de Chile, Subsecretaria de Redes Asistenciales, Ministerio de Salud, Gobierno de
Chile, 2020a. Disponible en: https://www.minsal.cl/wp-content/ uploads/2020/05/03-abr-Circular-2-Racionalizacion-uso-EPP-encontexto-atencion-pacientes-durante-pandemia.pdf

Subsecretaria de Redes Asistenciales. Circular No005: Protocolo de Reprocesamiento de Respiradores Tipo N95, FFP2 u otros Equivalentes, para Atención Clínica en el Contexto de Pandemia COVID-19. Santiago de Chile, Subsecretaria de Redes Asistenciales, Ministerio de Salud, Gobierno de Chile, 2020b. Disponible en: https://www.minsal.cl/wp-content/uploads/2020/ 05/Circular-N5-del-29-de-abril-2020-Respiradores-tipoN95.pdf

Subsecretaría de Salud Pública. Orientaciones para Atención Odontológica en Fase IV COVID-19. Santiago de Chile, Subsecretaría de Salud Pública, Ministerio de Salud, Gobierno de Chile, 2020. Disponible en: https://diprece.minsal.cl/wp-content/ uploads/2020/03/ORIENTACIONES-ATENCIONODONTOLOGICAS-COVID-19-.pdf

Superintendencia de Salud. Manuales de Acreditación para Prestadores Institucionales de Salud. Santiago de Chile, Superintendencia de Salud Disponible, 2020. Disponible en: http:/ /www.supersalud.gob.cl/observatorio/671/w3-propertyvalue4737.html.

van Doremalen, N.; Bushmaker, T.; Morris, D. H.; Holbrook, M. G.; Gamble, A.; Williamson, B. N.; Tamin, A.; Harcourt, J. L.; Thornburg, N. J.; Gerber, S. I.; et al. Aerosol and surface stability of SARS-CoV-2 as compared with SARS-CoV-1. N. Engl. J. Med., 382(16):1564-7, 2020.

Dirección para correspondencia:

Prof. Dr. Ramón Fuentes

Facultad de Odontología

Universidad de La Frontera

Av. Francisco Salazar 01145

Temuco

CHILE

Email: ramon.fuentes@ufrontera.cl

Recibido : 12-05-2020

Aceptado: 14-05-2020 